\title{
Alteration of pulmonary structure by Pseudomonas aeruginosa exoenzyme S
}

\author{
D. E. WOODS, W. S. HWANG,* M. S. SHAHRABADI and J. U. QUE
}

Department of Microbiology and Infectious Diseases and "Department of Pathology, University of Calgary Health Sciences Centre, Calgary, Alberta, Canada T2N 4N1

\begin{abstract}
Summary. Intratracheal administration of purified Pseudomonas aeruginosa exoenzyme $S$ elicited extensive, grossly observable damage in the rat lung within $2 \mathrm{~h}$. Light and electronmicroscopy revealed injury and necrosis of bronchial epithelium, type I pneumocytes and capillary endothelial cells after $1 \mathrm{~h}$; associated haemorrhage, fibrinous exudation and released type II cell lamellar bodies in alveolar lumina after 1-12 $\mathrm{h}$; progressively increasing accumulations of polymorphonuclear leucocytes in the bronchi and alveoli and in alveolar septae (interstitial pneumonia) after 1-12 $\mathrm{h}$; collapse of alveolar septal connective tissue and damage to pulmonary arterioles and venules. Treatment of monolayer cultures of bronchial fibroblasts with purified exoenzyme $\mathrm{S}$ elicited vacuolation of the cells with apparent membrane damage as revealed by light and electronmicroscopy. In-vivo production and activity of $P$. aeruginosa exoenzyme $\mathrm{S}$ may be an important pathogenicity determinant in the necrotising lung injury characteristic of $P$. aeruginosa pneumonia.
\end{abstract}

\section{Introduction}

A variety of specific and non-specific defence mechanisms serve to protect the lung from injury by micro-organisms and their products. These include mucociliary clearance, alveolar macrophages, humoral and cell-mediated immunity (Green, 1970). In spite of these usually efficient defence mechanisms, bacterial lung infections do occur. Over the past three decades, gram-negative micro-organisms have become increasingly important as causative agents of serious infections, particularly in hospitalised patients (Centers for Disease Control, 1984). Pseudomonas aeruginosa has become especially prominent in this regard (Renner et al., 1972).

Pulmonary infections with $P$. aeruginosa may occur in clinical settings other than nosocomial pneumonias, including community-acquired acute pneumonia (Sullivan et al., 1972), and in chronic lung infections of cystic fibrosis (CF) patients (Reynolds et al., 1976). Pulmonary infection with $P$. aeruginosa may be accompanied by severe tissue damage because this organism produces several virulence factors in mammalian hosts and these contribute to the pulmonary tissue damage (Woods

Received 17 Aug. 1987; accepted 24 Nov. 1987.

* Correspondence should be sent to W. S. Hwang. and Sokol, 1986). In addition to cell-associated factors, $P$. aeruginosa produces a number of exoenzymes, including exotoxin A, proteolytic enzymes, phospholipase $C$, and exoenzyme $S$, each of which has been implicated as a virulence determinant (Woods and Iglewski, 1983).

Histopathological descriptions of lung injury in acute $P$. aeruginosa infections have varied, depending upon the patient and how the infection was acquired. The lesions seen in nosocomially acquired necrotising pneumonia are characterised by alveolar septal necrosis with necrosis of arterial walls and secondary thrombosis. The alveolar exudate is predominantly mononuclear with some polymorphonuclear leucocytes (Mays et al., 1969). The pathology of community-acquired pneumonia caused by $P$. aeruginosa is characterised by multiple small abscesses and marked alveolar cell necrosis. Arterial wall necrosis, perivascular infiltrate and thrombosis were not noted (Tillotson and Lerner, 1968). The principal microscopic finding in cases of $P$. aeruginosa pneumonia acquired after bacteraemia originating from another site is intra-alveolar haemorrhage with patzhy alveolar septal necrosis (Fetzer et al., 1967). The pulmonary histopathology of chronic $P$. aeruginosa infections as seen in cystic fibrosis is dominated by bronchial changes including epithelial metaplasia with loss of cilia, predominance of mucous over serous acini in hyperplastic 
bronchial glands, goblet cell hyperplasia, acute and chronic inflammatory infiltrates, bronchiectasis and mucopurulent plugging of the airways (Bedrossian et al., 1976).

We have proposed that these various pathological pictures might be explained by infection with strains of differing exoproduct phenotypes (Que and Woods, 1987). Indeed, clinical isolates of $P$. aeruginosa are of significantly different exoproduct phenotypes depending upon their site of isolation, indicating that the host environment may select for the phenotypic expression of certain exoproducts in specific infections (Woods et al., 1986). Based upon these studies, we have concluded that elastase and exoenzyme $\mathbf{S}$ may play important roles in pulmonary infections with $P$. aeruginosa. A similar conclusion has been reached by comparative studies in animal models of lung infections produced by $P$. aeruginosa parent strains and isogeneic mutants singly deficient in either elastase or exoenzyme S. The parent strains were shown to be more virulent (Woods et al., 1982; Blackwood et al., 1983; Nicas et al., 1985; Woods and Sokol, 1985).

An alternative approach to the study of the pathogenesis of $\boldsymbol{P}$. aeruginosa pulmonary infection was that of Cash et al. (1982) who instilled purified $P$. aeruginosa exoproducts into the lungs of rats and demonstrated that the resulting pulmonary histopathology was similar to that induced by experimental infection with $P$. aeruginosa. Similarly, Gray and Kreger (1979) demonstrated that instillation of purified $P$. aeruginosa proteases into the lungs of rabbits produced changes not unlike those seen in some cases of human pneumonia. The present study was designed to examine the effects of direct instillation of purified $P$. aeruginosa exoenzyme $\mathrm{S}$ into rat lungs in order to determine whether the histopathological changes produced by exoenzyme $S$ could be correlated with any of the various pathological pictures described for $P$. aeruginosa pulmonary infections.

\section{Materials and methods}

\section{Exoenzyme S preparation}

Exoenzyme $\mathrm{S}$ was purified from culture supernates of $P$. aeruginosa strain DG1 grown at $32^{\circ} \mathrm{C}$ in mineral salts medium containing $110 \mathrm{~mm}$ succinate, $100 \mathrm{~mm}$ monosodium glutamate, glycerol $1 \%$ and $10 \mathrm{~mm}$ EDTA until late exponential phase (Woods and Que, 1987). Purification was achieved by sequential ammonium sulphate precipitation, DEAE-Sephacel anion exchange chromatography, acetone precipitation in the presence of $1 \mathrm{M} \mathrm{NaCl}$, and G100 Superfine gel filtration chromatography. Exoenzyme $\mathrm{S}$ was monitored during purification by an assay for ADP-ribosyl transferase activity, mouse and tissue culture cell toxicity and sodium dodecyl sulphate-polyacrylamide gel electrophoresis (Woods and Que, 1987).

\section{Instillation of purified exoenzyme $S$ into rat lungs}

Filter-sterilised $(0 \cdot 2-\mu \mathrm{m}$ pore size) exoenzyme $S$ preparations were diluted with sterile phosphatebuffered saline (PBS, $\mathrm{pH} \mathrm{7.0)}$ to yield solutions containing $(0.1 \mathrm{ml}) 100,50$ and $1 \mu \mathrm{g}$ of exoenzyme $\mathrm{S}$ per $0.1 \mathrm{ml}$. A tracheostomy was performed on young adult male Sprague-Dawley rats (200-220 g; Charles River, Canada) under ether anaesthesia and $0.1 \mathrm{ml}$ of active exoenzyme $\mathrm{S}$ or heat-inactivated exoenzyme $\mathrm{S}\left(100^{\circ} \mathrm{C}, 30 \mathrm{~min}\right.$; control) was deposited into a distal bronchus via a bead-tipped curved needle (Cash et al., 1979). Six animals from each group (active versus inactive exoenzyme $S, 3$ separate concentrations each) were killed by exsanguination under ether anaesthesia at intervals of 1 , 2,4 and $12 \mathrm{~h}$ after instillation.

\section{Tissue-culture monolayer treatment}

Dulbecco's Modified Eagle's Medium (DMEM; Flow Laboratories, Mississauga, Ontario) with sodium pyruvate $110 \mathrm{mg} / \mathrm{L}$ was supplemented with glutamine $584 \mathrm{mg} / \mathrm{L}$, penicillin $50 \mathrm{IU} / \mathrm{ml}$, streptomycin $50 \mu \mathrm{g} / \mathrm{ml}$, and fetal calf serum (Myoclone, Gibco, Burlington, Ontario) 10\%. Bronchial fibroblasts (CCD 14Br) were obtained from the American Type Culture Collection (Rockville, MD). Tissue-culture monolayers were harvested by rinsing with $5 \mathrm{ml}$ of trypsin-EDTA (Gibco Canada, Inc.), incubation for $10 \mathrm{~min}$ with $5 \mathrm{ml}$ of trypsinEDTA at $37^{\circ} \mathrm{C}$ in $\mathrm{CO}_{2} 7 \%$, mixing with an equal volume of cold medium and centrifugation $(5000 \mathrm{~g}$ for $10 \mathrm{~min}$ ). The cells were resuspended in fresh medium to a concentration of $1 \times 10^{5}$ cells $/ \mathrm{ml}$; $200 \mu \mathrm{l}$ were dispensed into each well of a microtitration plate and incubated overnight at $37^{\circ} \mathrm{C}$ in $\mathrm{CO}_{2}$ $7 \%$. Various concentrations of active or heatinactivated exoenzyme $S$ were filter sterilised, diluted in DMEM and added to the cells.

\section{Light and electronmicroscopy}

The lungs and heart were removed en bloc from control and exoenzyme S-treated animals, and onethird of the samples were fixed uninflated in buffered formalin (pH 7.0) 10\%. Another one-third of the samples were perfused through the trachea 
with buffered formalin (pH 7.0) $10 \%$ under $10 \mathrm{~cm}$ hydrostatic pressure. All of the above samples were fixed for a minimum of $24 \mathrm{~h}$ after which sagittal sections of lungs were embedded in plastic (glycolmethylacrylate) for light microscopic histochemical studies. Specimens to be examined by electronmicroscopy (final one-third) were cut into $1-\mathrm{mm}$ cubes and fixed in gluteraldehyde $4 \%$ in sodium cacodylate buffer $(0.05 \mathrm{M}, \mathrm{pH} 7.4)$ for $12 \mathrm{~h}$.

Plastic sections $(1 \mu \mathrm{m})$ of tissues fixed in the uninflated state were stained with haematoxylin and eosin ( $\mathrm{H} \& \mathrm{E})$, Giemsa, and a combined periodic acid-Schiff-silver stain (Preece, 1972). Plastic sections of tissues fixed in the inflated state were stained with $\mathrm{H} \& \mathrm{E}$ and reticulocyte staining to examine for alveolar septal loss (Woods et al., 1982).

Glutaraldehyde-fixed tissues were washed with cacodylate buffer, postfixed in osmium tetroxide $1 \%$ in cacodylate buffer for $2 \mathrm{~h}$, washed with cacodylate buffer, and dehydrated in increasing concentrations of acetone. Specimens were further dehydrated in propylene oxide and embedded in Epon 812 . Thick sections $(1 \mu \mathrm{m})$ were stained with toluidene blue and were examined by light microscopy to evaluate embedding and condition of tissues and to select areas for subsequent electronmicroscopic examination. Thin sections $(80 \mathrm{~nm})$ were stained with uranyl acetate and lead citrate. Thin sections were examined in a Hitachi $\mathrm{H}-600$ electronmicroscope.

Light microscopic examination of tissue-culture cells (bronchial fibroblasts, CCD 14Br) was performed directly in microtitration wells after staining with crystal violet. Tissue-culture monolayers of cells treated with active exoenzyme $S$ and heatinactivated exoenzyme $S$ (control) were rinsed three times with PBS $(200 \mu \mathrm{l})$, stained with crystal violet $(1 \%$ in PBS), and washed three further times with PBS to remove excess stain.

Tissue-culture cells from exoenzyme S-treated and control monolayers to be examined by electronmicroscopy were rinsed three times with PBS to remove tissue-culture medium. The monolayer was disrupted by scraping with a "rubber policeman" in the presence of PBS and the cells were transferred to a microfuge tube and pelleted by centrifugation (10000 g, $5 \mathrm{~min}$; Beckman Microfuge). Cells were fixed in glutaraldehyde $3 \%$ in cacodylate buffer $(0.05 \mathrm{M}, \mathrm{pH} 7.2)$ for $3 \mathrm{~h}$ at $4^{\circ} \mathrm{C}$. The cells were washed three times with cacodylate buffer and left in this buffer overnight. Cells were post-fixed in osmium tetroxide $1 \%$ at room temperature for $3 \mathrm{~h}$. Dehydration was with increasing concentrations of ethanol and the cells were embedded in Epon 812.
Thin sections $(80 \mathrm{~nm})$ were stained with uranyl acetate $0.5 \%$ in methanol followed by lead citrate $1 \%$ and examined in a Phillips 400 electronmicroscope.

\section{Results}

Intratracheal instillation of heat-inactivated control preparations did not produce gross or microscopic alterations in pulmonary structure of rats at any of the time points examined. All the rats given active exoenzyme $\mathbf{S}$ preparations at concentrations of $100 \mu \mathrm{g}$ and $50 \mu \mathrm{g}$ died within $6 \mathrm{~h}$ of instillation. The most notable symptom at the time of death was marked respiratory distress. The two highest doses of exoenzyme $\mathrm{S}(100$ and $50 \mu \mathrm{g})$ produced numerous, grossly observable confluent haemorrhagic lesions after $1 \mathrm{~h}$ and the lesions increased in size progressively and reached maximum size after $4 \mathrm{~h}$. Sagittal lung sections revealed that lesions extended throughout the depth of the lung. In additional experiments, animals were given $1 \mu \mathrm{g}$ of exoenzyme $S$ and allowed to live for 7 days. Lung structure failed to return to normal in any of the animals examined during this time period (data not shown).

Light microscopy of lungs from animals given intratracheal injections of $50 \mu \mathrm{g}$ or more of active exoenzyme $S$ revealed almost immediate reactive changes. Airways and parenchyma were completely obscured by the inflammatory response after $1 \mathrm{~h}$ (fig. 1). The following histopathological descriptions apply to lungs after instillation of $1 \mu \mathrm{g}$ of exoenzyme $\mathrm{S}$.

One hour after injection, injury to bronchial epithelium was evident (fig. 2). Increasing accumulations of inflammatory cells and fibrinous exudation, both intrabronchially and peribronchially, continued throughout the 12 -h time period until the bronchial epithelial layer was destroyed (fig. 3). Alveolar type-I epithelial cells and capillary endothelial cells showed progressive injury and necrosis from $1 \mathrm{~h}$ to $12 \mathrm{~h}$ after injection. Electronmicroscopic examination revealed consistent and obvious membrane damage to alveolar type-I epithelial cells. Fig. 4 shows the "lifting-off" of the membrane consistently observed in this cell type from $4 \mathrm{~h}$ to $12 \mathrm{~h}$ after injection.

Alveolar type-II epithelial cells showed hyperplasia $4 \mathrm{~h}$ after injection and this increased progressively throughout the study period. During this time, there was a marked increase in the releases of type-II cell lamellar bodies into alveolar lumina. Membrane damage was also a consistent finding in 


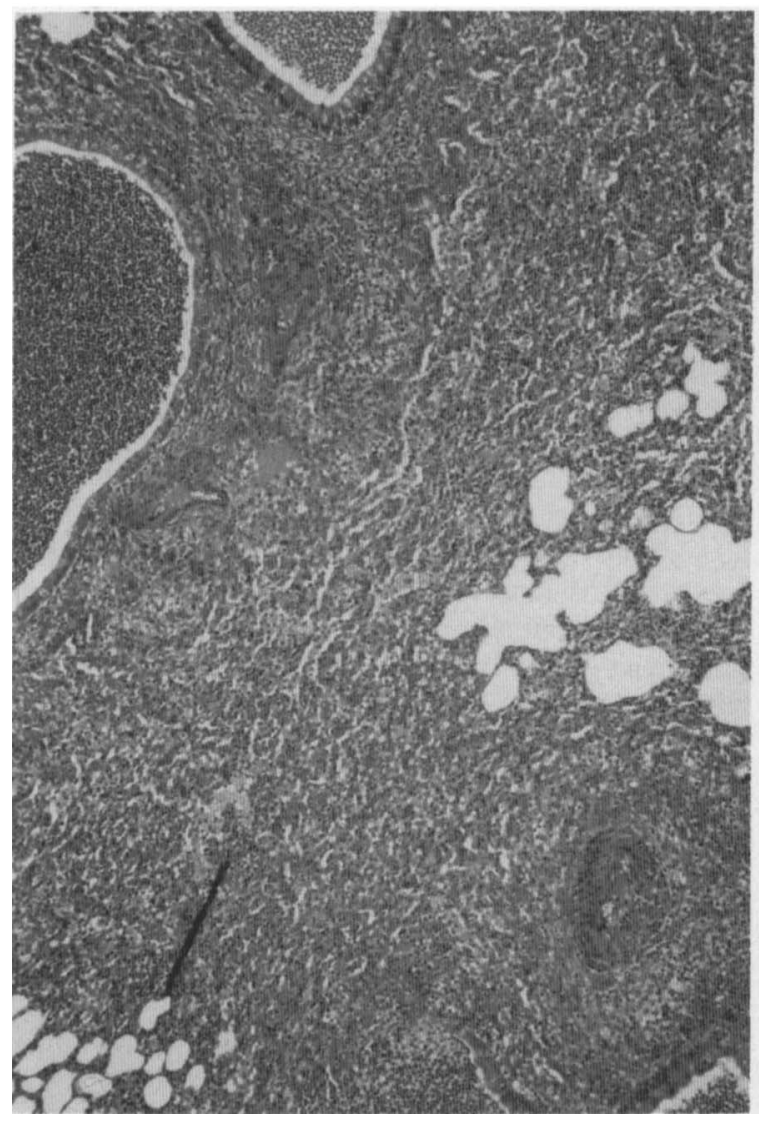

Fig. 1. H \& E stained section of rat lung $1 \mathrm{~h}$ after treatment with $100 \mu \mathrm{g}$ of purified exoenzyme S. Airways and lung parenchyma are totally obscured by suppurative inflammation consisting of polymorphonuclear leucocytes and proteinaceous exudation. $\times 50$.

this cell type after $12 \mathrm{~h}$; fig. 5 shows the vacuolation seen in type-II epithelial cells at this time.

Progressively increased accumulations of polymorphonuclear leucocytes occurred in bronchial and alveolar lumina as well as in alveolar septae (interstitial pneumonia) from $1 \mathrm{~h}$ to $12 \mathrm{~h}$ after injection. Toluidine blue staining of exoenzyme Streated lung tissue revealed vacuolation of pulmonary parenchyma reminiscent of that seen in typeII epithelial cells (fig. 6). Collapse of alveolar septal connective tissue and damage to pulmonary arterioles and venules was revealed by reticulocyte staining (fig. 7).

Treatment of monolayer cultures of bronchial fibroblasts with purified exoenzyme $S(100 \mathrm{ng})$ elicited vacuolation of the cells with apparent membrane damage $12 \mathrm{~h}$ after treatment (fig. 8). At $24 \mathrm{~h}$ after treatment, the monolayer was completely destroyed (fig. 9). Electronmicroscopic examination

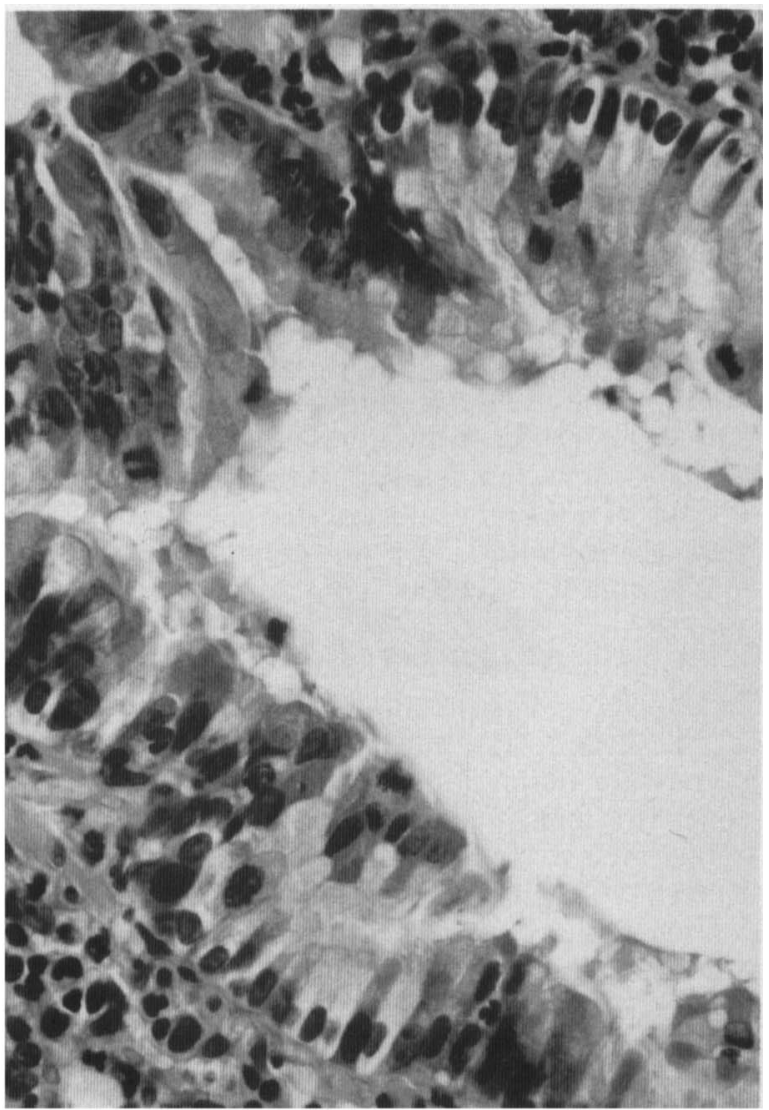

Fig. 2. H \& E stained section of rat lung $1 \mathrm{~h}$ after treatment with $1 \mu \mathrm{g}$ of purified exoenzyme S. Initial stages of airway injury are indicated by the significant alterations in bronchial epithelium. Initial stages of peribronchial inflammatory cell (polymorphonuclear leucocytes primarily) infiltration are also apparent. $\times 400$.

of bronchial fibroblasts at 12 and $24 \mathrm{~h}$ after treatment with exoenzyme $S$ demonstrated progressive vacuolation of cells from $12 \mathrm{~h}$ (fig. 10) to $24 \mathrm{~h}$ (fig. 11).

\section{Discussion}

$P$. aeruginosa produces several extracellular products which have been proposed as virulence determinants in pulmonary infections with this organism (Woods and Iglewski, 1983). Included among these are two distinct ADP-ribosyl transferases-exotoxin $\mathrm{A}$ and exoenzyme $\mathrm{S}$-which differ in their molecular structure and substrate specificity (Iglewski et al., 1977, 1978). Recent evidence from several studies indicates that exoenzyme $S$ may play a significant role in pulmonary infection in man. In animal model studies comparing infections produced by parental $P$. aeruginosa strains with 


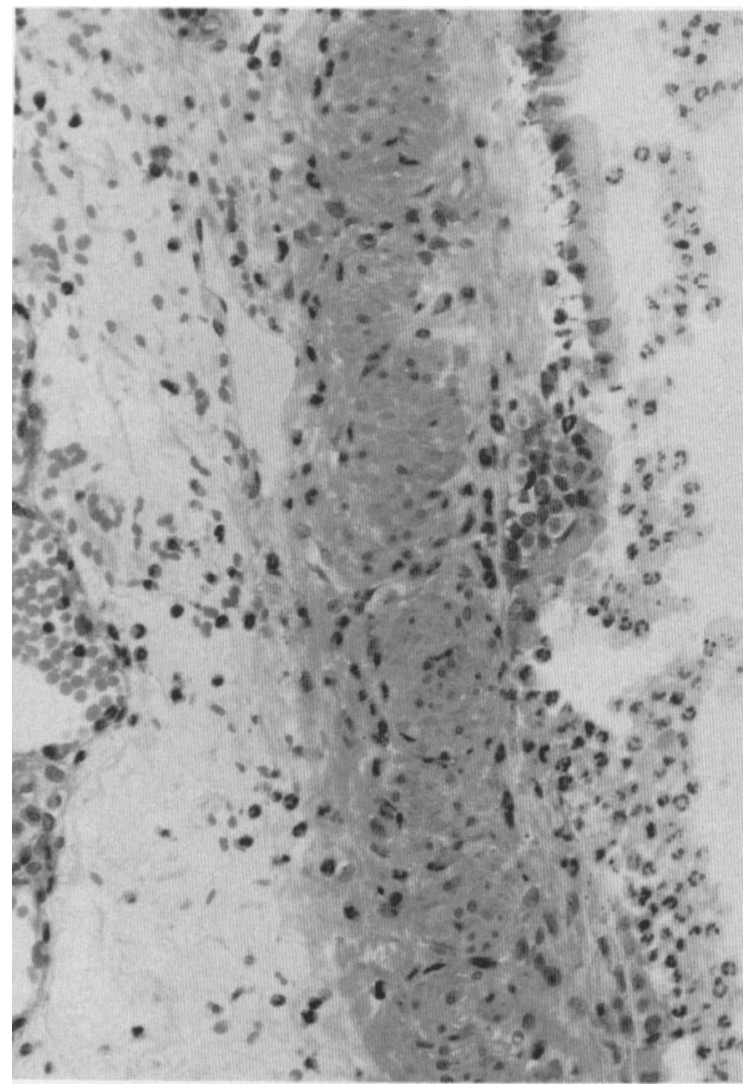

Fig. 3. $\mathrm{H} \& \mathrm{E}$ stained section of rat lung $12 \mathrm{~h}$ after instillation of $1 \mu \mathrm{g}$ of purified exoenzyme $\mathrm{S}$. There are accumulations of inflammatory cells and fibrinous exudation, both intrabronchially and peribronchially with destruction of the bronchial epithelial layer. $\times 100$.

those produced by isogeneic mutants, singly deficient in exoenzyme $S$, it has been shown that infections with exoenzyme S-positive strains are much more severe (Nicas et al., 1985; Woods and Sokol, 1985). Phenotypic analysis of $P$. aeruginosa strains isolated from pulmonary infections in man showed that these strains produce significantly more exoenzyme $\mathrm{S}$ than strains isolated from other clinical sources such as urine or wound infections (Woods et al., 1986). Further evidence that exoenzyme $S$ may play a role in lung injury during $P$. aeruginosa infections is provided by the present study, which is the first light and electronmicroscopic characterisation of lung injury after intratracheal instillation of purified exoenzyme $S$.

Descriptions of the pathology associated with $P$. aeruginosa lung infections have varied depending upon the patient or upon how the infection was acquired. The aim of the present study was to determine the relationship of those structural

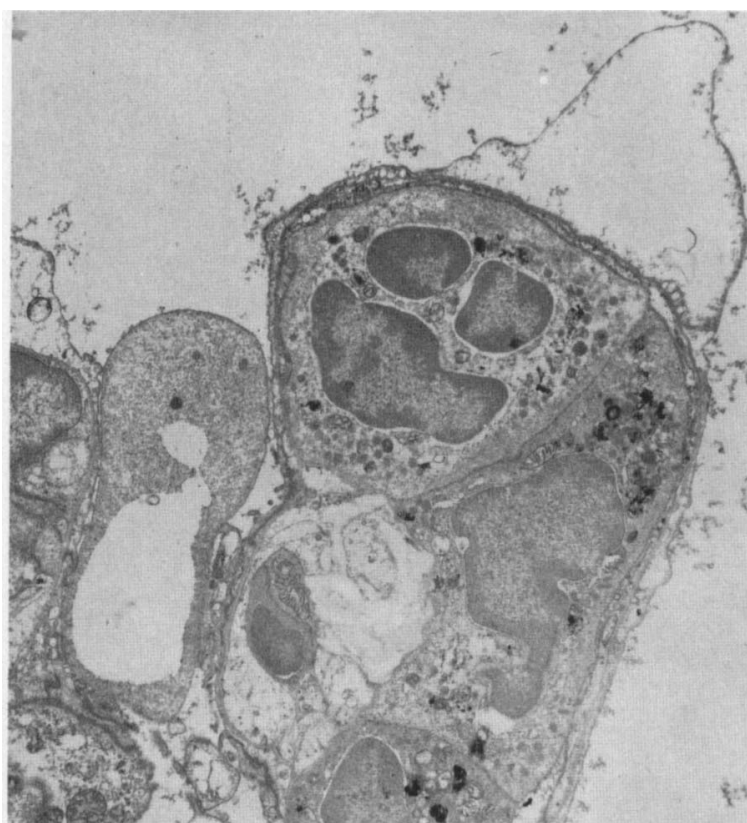

Fig. 4. Electronmicrograph of alveolar type-I epithelial cell in lung $4 \mathrm{~h}$ after instillation of $1 \mu \mathrm{g}$ of purified exoenzyme $\mathrm{S}$. There is apparent membrane damage characterised by "lifting-off" of the membrane. Uranyl acetate and lead citrate stained, $\times 5000$.

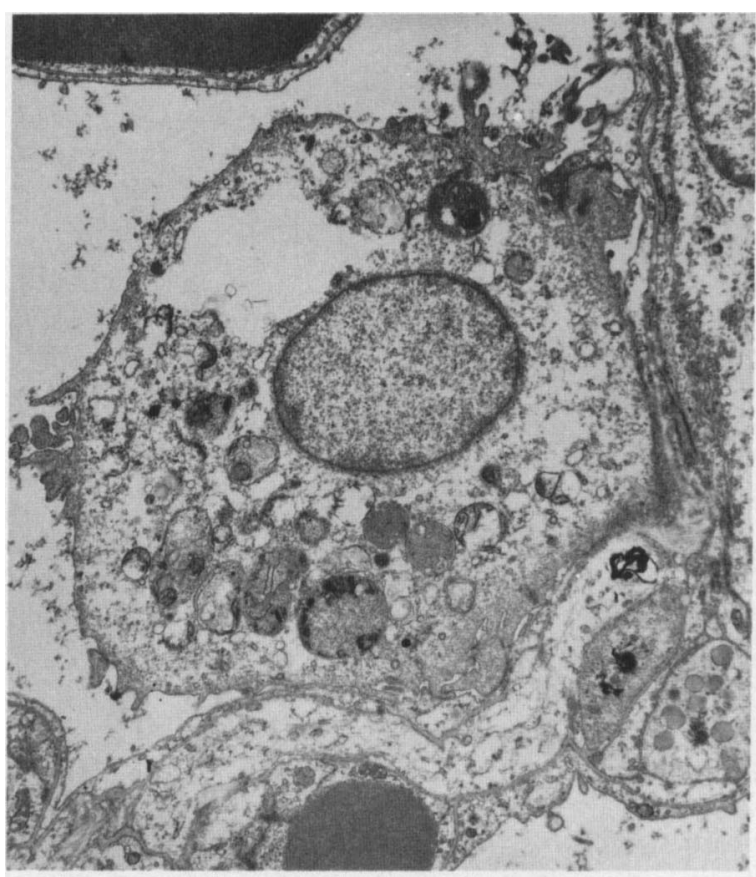

Fig. 5. Electronmicrograph of alveolar type-II epithelial cell in lung $4 \mathrm{~h}$ after instillation of $1 \mu \mathrm{g}$ of purified exoenzyme $\mathrm{S}$. There is vacuolation and lamellar body inclusion and release into alveolar lumina. Uranyl acetate and lead citrate stained, $\times 6000$. 


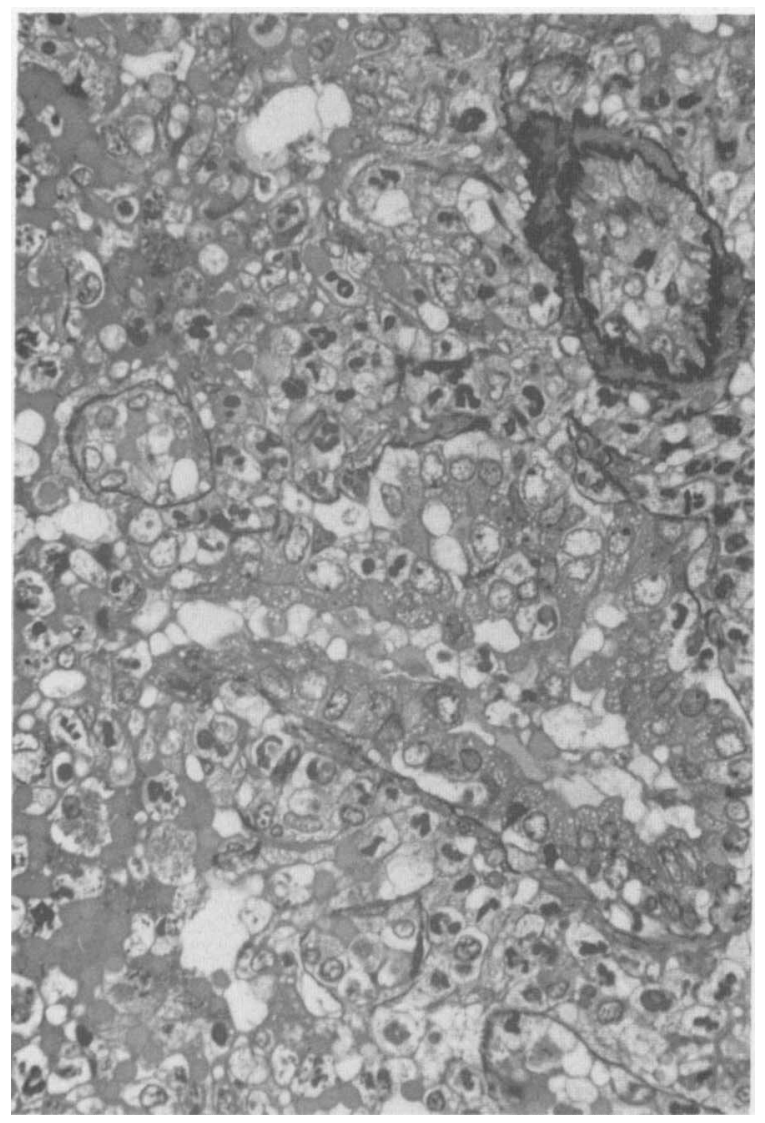

Fig. 6. Toluidine blue stained section of rat lung $12 \mathrm{~h}$ after instillation of $1 \mu \mathrm{g}$ of purified exoenzyme $\mathrm{S}$. There is accumulation of polymorphonuclear leucocytes in bronchial and alveolar lumina as well as in alveolar septae (interstitial pneumonia). Vacuolation of pulmonary parenchyma is reminiscent of that seen in alveolar type-II epithelial cells. $\times 50$.

changes induced in lungs by instillation of exoenzyme $\mathrm{S}$ with the histopathological changes described for nosocomial, community-acquired, or bacteraemic $\boldsymbol{P}$. aeruginosa pneumonias, as well as those described for chronic $P$. aeruginosa lung infections in patients with cystic fibrosis. Surprisingly, the histopathological changes seen in rat lungs after instillation with purified exoenzyme $S$ appeared to overlap with all of the various histopathological descriptions previously published for $P$. aeruginosa lung infections, regardless of origin.

Alveolar septal necrosis, necrosis of arterial walls, and secondary vessel thrombosis were the morphologic features stressed in the diagnosis of gramnegative bacillary necrotising pneumonias induced by the inhalation of aerosolised $P$. aeruginos $a$ from positive-pressure nebulisers (Mays et al., 1969). The present study documents clearly that collapse of

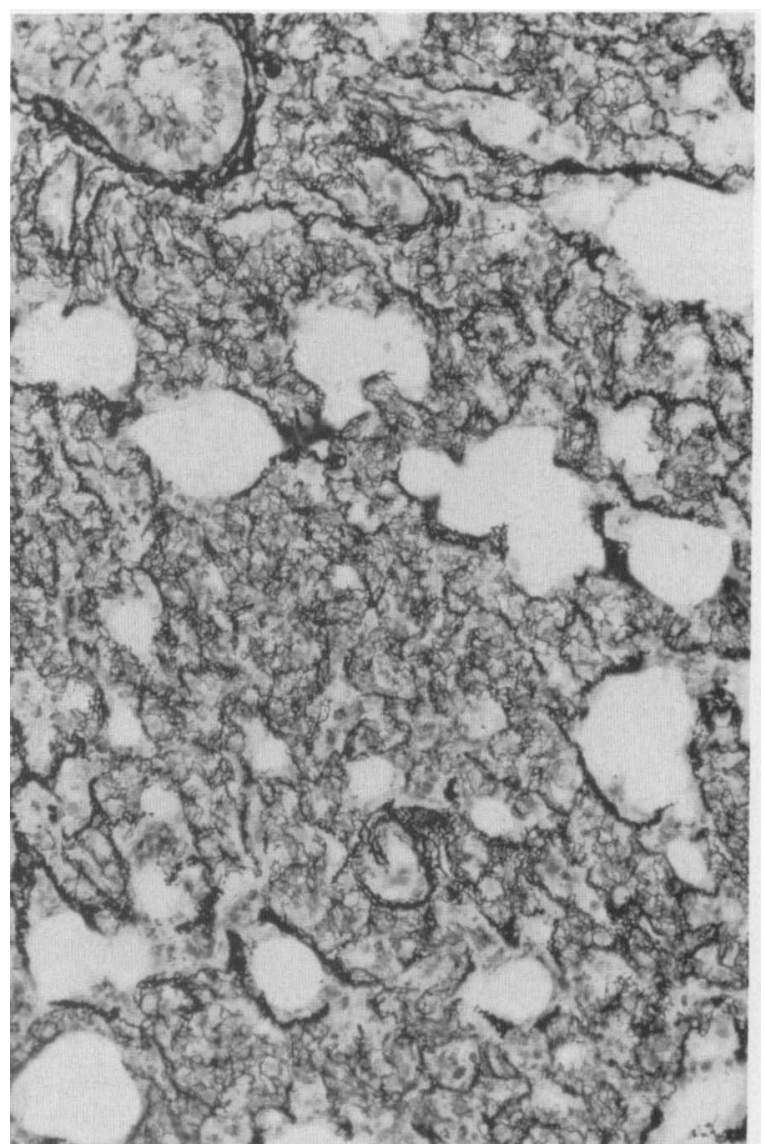

Fig. 7. Section of rat lung $1 \mathrm{~h}$ after instillation of $1 \mu \mathrm{g}$ of purified exoenzyme $\mathrm{S}$, showing collapse of alveolar septal connective tissue. Lungs had been fixed and inflated with formalin $10 \%$ in PBS under $10 \mathrm{~cm}$ hydrostatic pressure before embedding; reticulocyte staining; $\times 50$.

alveolar septal connective tissue and damage to pulmonary arterioles and venules, as revealed by reticulocyte staining of exoenzyme S-treated lungs, is a basic feature of lung injury caused by exoenzyme S. Thus, histopathological changes during nosocomial pneumonia caused by $P$. aeruginosa could be explained partially by the elaboration of exoenzyme $S$ in vivo during these infections.

Tillotson and Lerner (1968) described the pathology of ten cases of $P$. aeruginosa pneumonia that were neither the result of a pre-existing bacteraemia, nor nosocomially acquired. Multiple small abscesses were grossly present and marked alveolar cell-wall necrosis was characteristic at microscopic examination. Arterial wall necrosis, perivascular infiltration, and thrombosis were not noted. Similar, though not identical, pathological findings were seen after instillation of purified exoenzyme $S$ into 


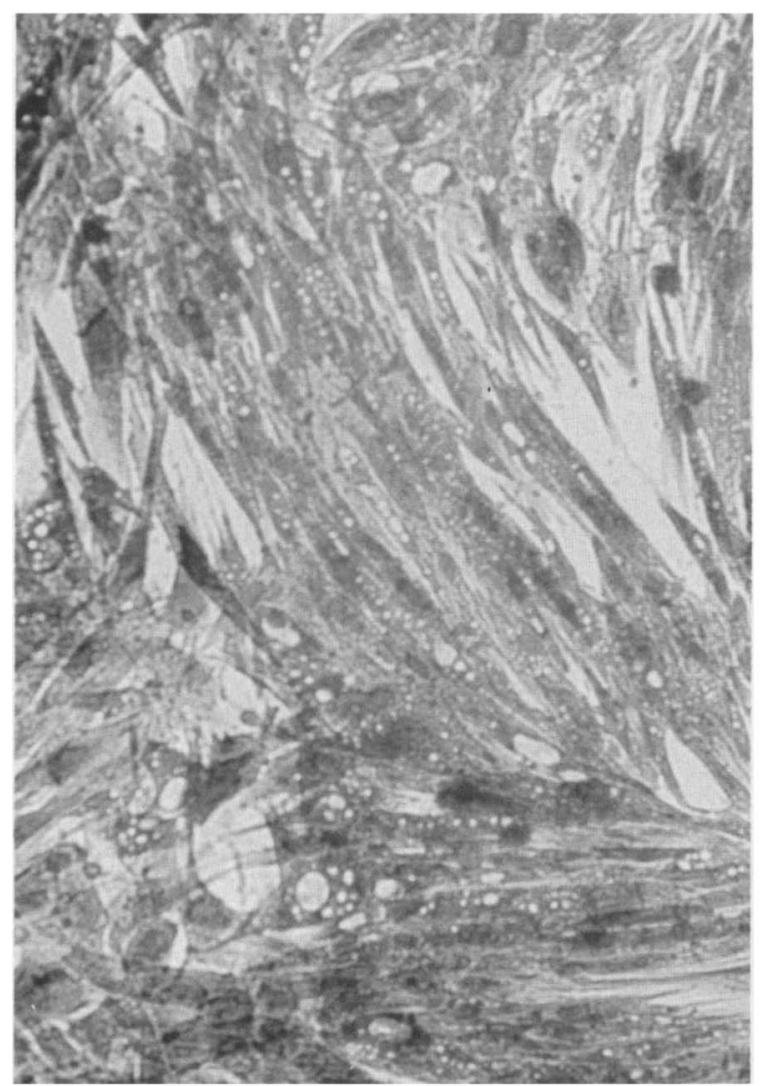

Fig. 8. Monolayer culture of bronchial fibroblasts $12 \mathrm{~h}$ after treatment with $100 \mathrm{ng}$ of purified exoenzyme S, showing apparent membrane damage (vacuolation). Crystal violet stained, $\times 400$.

the lungs of experimental animals. In comparing our results with the descriptions of Tillotson and Lerner (1968), the differences may not be only in the degree of injury but there may be qualitative differences.

Similar to the findings reported for nosocomial $P$. aeruginosa pneumonias (Mays et al., 1969), we have seen arterial-wall necrosis as a result of instillation of exoenzyme $S$ into the lungs of experimental animals. This is significantly different from the reports of Tillotson and Lerner (1968) and those of Fetzer et al. (1967) that in P. aeruginosa pneumonia originating from bacteraemia, organisms and necrosis occurred only in the capillaries. Arterial-wall necrosis was not seen when purified proteases were instilled into the lungs of rabbits (Gray and Kreger, 1979). Based upon these findings, it would appear that strains isolated from cases of nosocomial pneumonia should be examined for phenotypic expression of exoenzyme $\mathrm{S}$, or pulmonary secretions from these patients should be

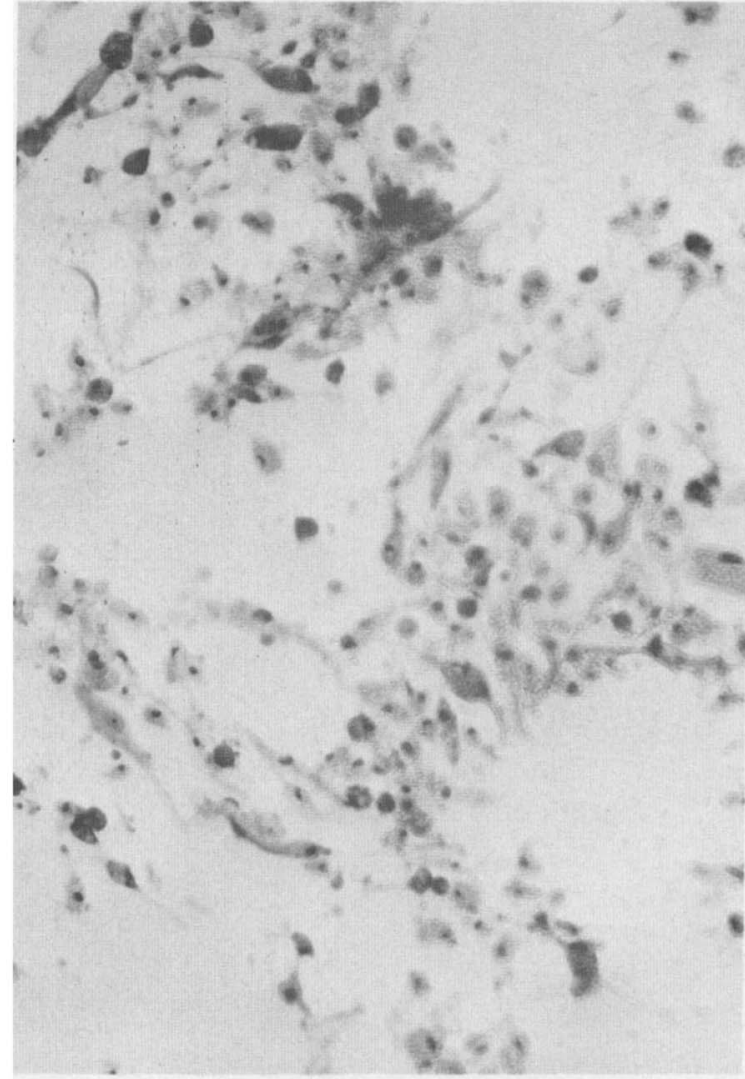

Fig. 9. Monolayer culture of bronchial fibroblasts $24 \mathrm{~h}$ after treatment with $100 \mathrm{ng}$ of purified exoenzyme S. Monolayer has been completely destroyed. Crystal violet stained, $\times 400$.

examined for the presence of exoenzyme S, or both should be done.

The pulmonary histopathology described for $P$. aeruginosa infections in cystic fibrosis is dominated by bronchial changes. These include epithelial metaplasia with loss of cilia, predominance of mucous versus serous acini in hyperplastic bronchial glands, goblet cell hyperplasia, acute and chronic inflammatory infiltrates, bronchiectasis and mucupurulent plugging of the airways (Bedrossian et al., 1976). The earliest changes noted after instillation of exoenzyme $\mathrm{S}$ into rat lungs were disruption of bronchial epithelium and airway inflammation. Additional pathological changes in airways were not seen in our studies, presumably because of the acute nature of the experiments. It is conceivable that chronic exposure to exoenzyme $\mathrm{S}$, as might occur during chronic $P$. aeruginosa infections, could lead to these changes.

It is interesting to note that the most consistent morphologic alteration seen in individual cells of 


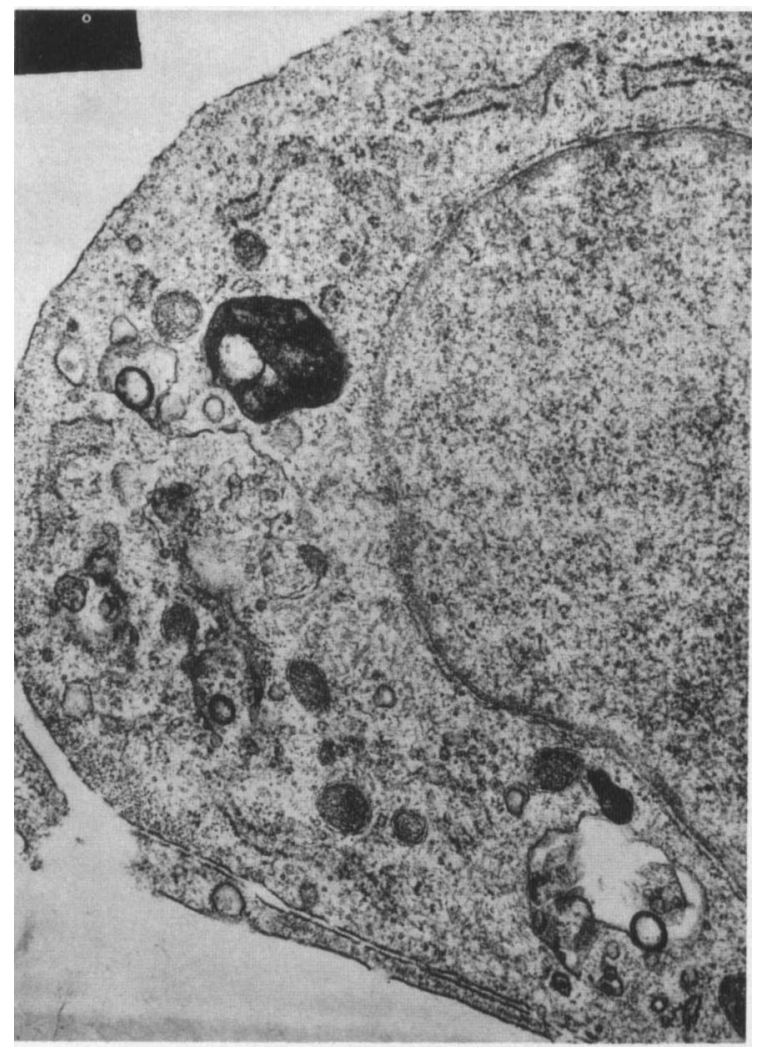

Fig. 10. Electronmicrograph of bronchial fibroblast $12 \mathrm{~h}$ after treatment with $100 \mathrm{ng}$ of purified exoenzyme $S$. Early membrane damage as indicated by vacuolation is apparent. Uranyl acetate and lead citrate stained, $\times 5000$.

both lung tissue and tissue-culture monolayers exposed to exoenzyme $S$ was an apparent membrane disruption which led either to "lifting off" of the membrane or vacuolation. This finding is in agreement with some of our recent studies in which we have demonstrated that exoenzyme $S$ acts at the level of the cytoplasmic membrane (Que, J. U. and Woods, D. E., unpublished observations).

There is little question that the virulence of $P$. aeruginosa is multifactorial. However, results from phenotypic analyses of $P$. aeruginosa strains isolated from a variety of infection sites, indicate that

\section{REFERENCES}

Bedrossian C W M, Greenberg S D, Singer D B, Hansen J J, Rosenberg H S 1976 The lung in cystic fibrosis: a quantitative study including prevalence of pathologic findings among different age groups. Human Pathology 7:195-204.

Blackwood L L, Stone R M, Iglewski B H, Pennington J E 1983 Evaluation of Pseudomonas aeruginosa exotoxin $\mathrm{A}$ and

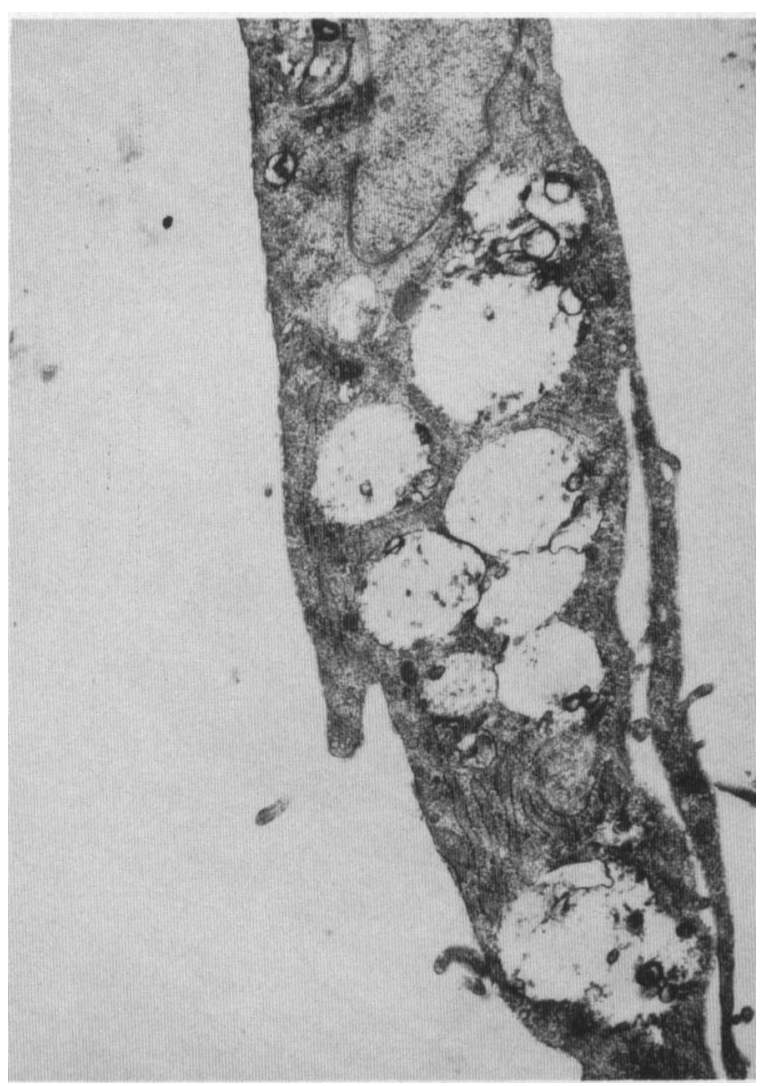

Fig. 11. Electronmicrograph of bronchial fibroblast $24 \mathrm{~h}$ after treatment with $100 \mathrm{ng}$ of purified exoenzyme S. Cell destruction by excessive membrane vacuolation. Uranyl acetate and lead citrate stained, $\times 2000$.

certain exoproducts may play a more important role in certain types of infections (Woods et al., 1986). This hypothesis is confirmed by the present studies which indicate that the pathological changes described for $P$. aeruginosa lung infections in a variety of clinical settings may be reproduced by a single $P$. aeruginosa exoproduct, exoenzyme $S$.

This work was supported by the Medical Research Council of Canada. Excellent technical assistance was provided by Mary McLellan and Marina To. D.E.W. is an Alberta Heritage Foundation for Medical Research Scholar.

elastase as virulence factors in acute lung infection. Infection and Immunity 39: 198-201.

Cash H A, Woods D E, McCullough B, Johanson W G, Bass J A 1979 A rat model of chronic respiratory infection with Pseudomonas aeruginosa. American Review of Respiratory Diseases 119:453-459.

Cash H A, Straus D C, Bass J A 1982 Pseudomonas aeruginosa exoproducts as pulmonary virulence factors. Canadian Journal of Microbiology $29: 448-456$. 
Centres for Disease Control 1984 National nosocomial infection study report: annual summary 1982. Centers for Disease Control, Atlanta, GA.

Fetzer A E, Werner A S, Hagstrom J W C 1967 Pathologic features of pseudomonal pneumonia. American Review of Respiratory Diseases 96:1121-1130.

Gray L, Kreger A 1979 Microscopic characterization of rabbit lung damage produced by Pseudomonas aeruginosa proteases. Infection and Immunity 23: 150-159.

Green G M 1970 The J. Burns Amberson Lecture: In defense of the lung. American Review of Respiratory Diseases 102:691703.

Iglewski B H, Liu P V, Kabat D 1977 Mechanism of action of Pseudomonas aeruginosa exotoxin A: adenosine diphosphate-ribosylation of mammalian elongation factor 2 , in vitro and in vivo. Infection and Immunity 15:138-144.

Iglewski B H, Sadoff J, Bjorn M J, Maxwell E S 1978 Pseudomonas aeruginosa exoenzyme S: an adenosine diphosphate ribosyltransferase distinct from toxin A. Proceedings of the National Academy of Sciences of the USA 75: 3211-3215.

Mays B B, Thomas G D, Leonard J S, Pierce A K 1969 Gramnegative bacillary necrotizing pneumonia: a bacteriologic and histopathologic correlation. Journal of Infectious Diseases 120:687-697.

Nicas T I, Frank D W, Stenzel P, Lile J D, Iglewski B H 1985 Role of exoenzyme $\mathrm{S}$ in chronic Pseudomonas aeruginosa lung infections. European Journal Clinical Microbiology 4:175-179.

Preece A 1972 A manual for histologic technicians. 3rd edn. Little, Brown and Co., Boston.

Que J U, Woods D E 1987 Alteration of lung structure and function by Pseudomonas aeruginosa. Pathology and Immunopathology Research 6:93-102.
Renner R R, Coccaro A P, Heitzman E R, Dailey S, Markarian B 1972 Pseudomonas pneumonia: A prototype of hospitalbased infection. Radiology 105: 555-562.

Reynolds H Y, Disant'agnese P A, Zierdt C H 1976 Mucoid Pseudomonas aeruginosa: a sign of cystic fibrosis in young adults with chronic pulmonary disease? Journal of the American Medical Association 236:2190-2192

Sullivan R J, Dowdle W R, Marine W M, Hierholzer J C 1972 Adult pneumonia in a general hospital. Etiology and host risk factors. Archive of Internal Medicine 129: 935-942.

Tillotson J R, Lerner A M 1968 Characteristics of nonbacteremic Pseudomonas pneumonia. Annals of Internal Medicine 68:295-307.

Woods D E, Cryz S J, Friedman R L, Iglewski B H 1982 Contribution of toxin $\mathbf{A}$ and elastase to virulence of Pseudomonas aeruginosa in chronic lung infections of rats. Infection and Immunity 36:1223-1228.

Woods D E, Iglewski B H 1983 Toxins of Pseudomonas aeruginosa: new perspectives. Reviews of Infectious Diseases 5:S715-S722.

Woods D E, Sokol P A 1985 Use of transposon mutants to assess the role of exoenzyme $S$ in chronic pulmonary disease due to Pseudomonas aeruginosa. European Journal of Clinical Microbiology 4:163-169.

Woods D E, Sokol P A 1986 Role of Pseudomonas aeruginosa extracellular enzymes in lung disease. Clinical and Investigative Medicine 9: 108-112.

Woods D E, Que J U 1987 Purification of Pseudomonas aeruginosa exoenzyme S. Infection and Immunity 55:579586.

Woods D E, Schaffer M S, Rabin H R, Campbell G D, Sokol P A 1986 Phenotypic comparison of Pseudomonas aeruginosa strains isolated from a variety of clinical sites. Journal of Clinical Microbiology 24:260-264. 
